\title{
EXPLORING FEEDBACK AND ENDOGENEITY IN AGENT-BASED MODELS
}

\author{
Ignacio J. Martinez-Moyano \\ Charles M. Macal \\ Argonne National Laboratory \\ 9700 South Cass Avenue \\ Argonne, IL 60439, USA
}

\begin{abstract}
Agent-based modeling is an approach used to describe systems composed of autonomous, independent, interactive, and potentially adaptive agents. Although agent-based models (ABMs) often include endogenous relationships that exist in agent-level interactions, such relationships are seldom salient when the structural elements of the models are analyzed and communicated. There are close relationships between agent-based modeling and other systems modeling techniques, such as the system dynamics approach. In system dynamics, feedback effects among major model components that can rapidly take systems far from equilibrium states are central to the modeling approach. In this paper we distinguish between structural endogeneity and behavioral endogeneity in models and derive an explicit representation of feedback and endogeneity in agent models - agent feedback diagrams. Finally, we describe a way that endogeneity and feedback may be highlighted in agent-based modeling and simulation.
\end{abstract}

\section{FEEDBACK, ENDOGENEITY, AND SYSTEM STRUCTURE}

This paper deals with the concept of endogeneity in models, how to identify it and represent it. Specifically, the idea of the existence and representation of endogeneity in agent-based models (ABMs) is addressed. Since the concepts of endogeneity and feedback are central to system dynamics (Richardson 1991, Richardson and Pugh 1981, Richardson 2011) and because "system dynamics is much more than a simulation technique, it is a comprehensive structured approach to modeling a complex system" (North and Macal 2007, p. 66), in order to explore the concepts of endogeneity and feedback in agent-based models, ideas and insights from system dynamics modeling are employed.

Finding endogenous explanations of system behavior is at the core of the system dynamics approach (Forrester 1961, Richardson and Pugh 1981, Forrester 1958). Endogenous explanations (explanations arising from within) are explanations, or theories, that seek to generate and explain the dynamics of a system through the interaction of the variables represented in the model (Sterman 2000). Exogenous explanations (explanations arising from without) alternatively, are explanations, or theories, that seek to generate and explain the dynamics of a system through the use of variables whose behavior is completely assumed (as such, explanations are not generated within the boundary of the model or theory). Endogeneity is present in some agent-based models but, although it is recognized as important and critical in understanding the dynamic behavior exhibited in the systems under study, it is seldom communicated in a salient manner due to the traditional structural representation of such models (for an example, see Auchincloss and Diez Roux 2008). By explicitly representing endogenous mechanisms in their models, agent-based modelers may more easily communicate the underlying structural characteristics that generate model results and insights.

Endogeneity has been studied in the social sciences, in general, and in the econometrics literature specifically, and many times it has been conceptualized as a problem in models (Nakamura and Nakamura 1998, Antonakis et al. 2010). In such literature, the problems identified with endogeneity are predominantly connected to data-driven models, and the solutions posed are related to statistical manipulation and consistency. Our work differs from such an approach, as we are concerned with endogeneity in 


\section{Martinez-Moyano and Macal}

the context of causal models of different levels of detail of real systems in which current action is often based on the results of previous actions (i.e., feedback). In this case, accurate representation of causal processes takes precedence over statistical adequacy or exclusion. Therefore, we say that in models that represent complex systems, endogeneity and feedback are normal and desirable to increase the fidelity of representation of the real system. Also, due to the nature of endogeneity, they should not be evaluated using a dichotomous approach (exists, does not exist); instead, they should be conceptualized within a continuum with degrees of endogeneity in models.

An endogeneity continuum that has the potential to yield robust descriptions of endogeneity-robust in the sense of generating sensible explanations of behavior arising from within the boundaries of models or theories - may be characterized using different dimensions. One important dimension is the feedback dimension. Along this dimension, a continuum may start with non-feedback-based explanations (although still endogenous in a strict sense) and conclude with explanations based on feedback processes. We have identified endogeneity along this dimension as structural endogeneity.

Structural endogeneity then, on one side, and in the strictest sense, would be represented by variables that are computed within the model (endogenous to the model) but that are not part of any feedback process. Such variables, however, might just be a simple (even linear) transformation of exogenous parameters or variables, which would make the degree of endogeneity of such variables extremely low. Consider the simple food model expressed in Equations (1) through (3). In this model, three exogenous-outside of the model-variables (population, food per capita, and price of food) are used to compute two endogenous variables (food expenditure and food consumption). In this model, although food consumption and food expenditure are strictly endogenous (computed inside the boundary of the model), their behavior is a simple transformation of the exogenous variables they depend on, which makes their usefulness a function of the quality of the exogenous (completely assumed) variables used in the model. Furthermore, as food consumption is a function of two exogenous variables - population and food per capita, with simple algebra, food expenditure can be expressed as a function of the three exogenous parameters in the model (see Equation 4).

Food Expenditure $=$ Food Consumption $\times$ Price of Food

Food Consumption $=$ Population $\times$ Food Per Capita

Population, Food Per Capita, Price of Food $=$ constant

Food Expenditure $=$ Population $\times$ Food Per Capita $\times$ Price of Food

Alternatively, on the opposite side of the continuum, a variable might be a function of itself (and other variables) as part of feedback mechanisms, making the degree of structural endogeneity of such variable high. We are interested in identifying and highlighting structural-feedback-based - endogeneity in models. Therefore, in any model of a system, in order to be able to have feedback-based endogenous explanations of system behavior, feedback loops, "the basic structural element in systems" (Forrester 1968, p. 4-5) must exist so that the behavior of variables of interest can be traced back to themselves. Without closed feedback loops, and even with these present, the variations in behavior of the variables of interest could ultimately be traced back (potentially entirely) to variables outside the system (and therefore be traced back to unexplained, only assumed, phenomena). Although feedback loops "enable the [feedbackbased] endogenous point of view and give it structure" (Richardson 1991, p. 298), thinking about endogeneity needs to go beyond the structural dimension. An important second dimension identified-in addition to the structural dimension-in understanding degrees of endogeneity is the behavioral dimension.

Consider that, even when variables in a model exhibit high degrees of structural endogeneityfeedback-based, the dynamic behavior of such variables (due to their sources of variability) can come both from exogenous parameters and from internal feedback mechanisms present in the model. As the variables in the model are a function of other variables (that may, or may not, be a part of feedback mechanisms), their degree of behavioral endogeneity will be a function of the strength of influence of the dif- 
ferent feedback loops that the variables belong to (the gain of the loops) and the strengths of the associations that the variables have with exogenous parameters. Therefore, two types of endogeneity are identified, structural (or potential) endogeneity and behavioral (or actual) endogeneity of variables.

For example, consider a simple model with four variables (one exogenous and three endogenous) as described in Equations (5) through (8). In this small model, because of the relationships among the variables, two feedback loops are present, one between $x$ and $y$, and another one between $x$ and $z$.

$$
\begin{aligned}
& x=f(\text { exo, } y, z) \\
& y=f(x) \\
& z=f(x) \\
& \text { exo }=\text { constant }
\end{aligned}
$$

Although identifying the structural endogeneity of the variables in simple models might be straightforward, we describe it for pedagogical purposes. In the case of the model described in Equations (5) through (8), the structural endogeneity of variable $x$ is higher than that of variables $y$ and $z$, since $x$ is part of two feedback mechanisms in the model (100\% of all feedback mechanisms), while $y$ and $z$ are part of only one feedback mechanism each (50\% of all feedback mechanism present). For variables in this simple model, and more complex ones, graph theoretic approaches can potentially be used to identify structural endogeneity and to quantify its degree (for an example of the use of graph theory in the structural analysis of system dynamics models, see Oliva 2004).

However, the fact that $x$ is part of $100 \%$ of the feedback mechanisms present in the model does not mean that its dynamic behavior will be more influenced by the feedback processes (due to the size of the loop gains) than by the exogenous parameter influencing it (exo). Additionally, as $x$ is influenced by more than one feedback loop, it is unclear which feedback loop contributes more to its behavior over time (for a method to identify loop dominance, see Mojtahedzadeh, Andersen, and Richardson 2004). It could be the case that $x$, although influenced by the feedback processes that it shares with $y$ and $z$, behaves basically as a replica of the exogenous influence exo. If this were the case, we could say that, although feedback is present in the model, the explanation that the model is able to provide is basically a duplication of the exogenous parameters used. In the specific case of variable $x$, we could say that, although high on structural endogeneity, it would exhibit low behavioral endogeneity.

In order to make salient, clarify, and communicate the feedback structure of models (and their boundaries), the system dynamics approach offers tools such as model-boundary charts, causal-loop diagrams, and stock-and-flow diagrams. The use of such tools in agent-based modeling and simulation has the potential of helping communicate how structural endogeneity exists and interacts in such models. These tools are explained next.

\section{TOOLS}

\subsection{Model-boundary Chart}

Tools that can help identify and communicate feedback structure in models can also aid in the modelbuilding process. For example, by clarifying components of the model boundary through the use of a model-boundary chart, modelers can identify missing connections or interactions in the model.

A model-boundary chart reviews the scope of the model by presenting a list of the variables that are endogenous, exogenous, and excluded from the model. For example, assume a simple model of population growth as described in Equations (9) through (11).

$$
\begin{aligned}
& \text { population }=f(\text { births, deaths }) \\
& \text { births }=f(\text { population, fertility }) \\
& \text { deaths, fertility }=\text { constant }
\end{aligned}
$$




\section{Martinez-Moyano and Macal}

This simple model has two endogenous variables and two exogenous variables. In Table 1 we show the list of the model's endogenous, exogenous, and excluded variables. It is useful to have such a list as this allows the modeler to clearly understand and communicate the sources of change (internal or external) of the variables of interest in the model. In addition, by explicitly listing excluded variables, the modeler acknowledges the limits of the model, or theory, about the system. Although the list of excluded variables can be literally infinite, the idea is to list the variables that are excluded from the model and relevant to the behavior of interest. In the simple model of population growth presented here, mortality is relevant but not yet included in the model. By explicitly showing mortality in the model-boundary chart (Table 1), the modeler delimits the reach of the theory embodied in the model and creates a clear path for future development.

Table 1: Model-boundary Chart

\begin{tabular}{lll}
\hline Endogenous & Exogenous & Excluded \\
\hline Population & Deaths & Mortality \\
Births & Fertility & \\
\hline
\end{tabular}

\subsection{Causal-loop Diagrams}

Causal-loop diagrams are tools to depict and understand the causal structure underlying model design. Causal diagrams "consist of variables connected by arrows denoting the causal influence among the variables" (Sterman 2000, p. 138). Although, in general, causal diagrams can be both "open loop" and "closed loop," the closed loop version is the one that interests us because when the causal chain represented in the diagram creates a closed loop, feedback-based endogeneity is identified. Figure 1 is an example of an open-loop diagram followed by two closed-loop diagrams.

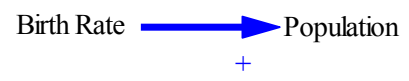

(a)

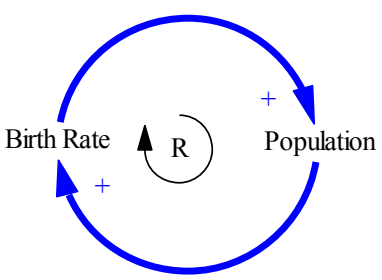

(b)

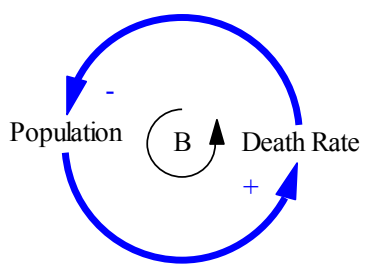

(c)

Figure 1: Causal Diagrams. (a) Open-loop Causal Chain. (b) Reinforcing/Positive Closed-loop Causal Chain. (c) Balancing/Negative Closed-loop Causal Chain.

In the diagrams shown in Figure 1, we present a simple hypothetical model of population dynamics. In the open-loop diagram (Figure 1a), we say that birth rate causally influences population. In the first closed-loop diagram (Figure 1b), we expand this thinking to say that while birth rate influences population, population, in turn, also causally influences birth rate. Finally, in the second closed-loop diagram (Figure 1c), we expand the theory to include that population is also causally linked to death rate and that, in turn, death rate influences population.

In casual diagrams, the variables are connected by arrows (causal links) with polarity signs denoting the type of influence that the variable at the beginning of the arrow has on the variable at the end of the arrow. Link polarities are meant to "describe the structure of the system" (Sterman 2000, p. 139), not the behavior of the variables in the causal chain. Arrows in causal connections indicate the direction of causality between variables. Polarity signs ("+" and "-") indicate the polarity of the relationship between the variables if there was a change. A positive sign ("+") means that, all else being equal, increases in the variable at the beginning of the arrow-the cause-will result in increases in the variable at the end of the ar- 
row - the effect - and that, all else being equal, decreases in the variable at the beginning of the arrow will result in decreases in the variable at the end of the arrow. Similarly, a negative ("-") sign means that, all else being equal, increases in the variable at the beginning of the arrow will result in decreases in the variable at the end of the arrow, and, similarly, that, all else being equal, decreases in the variable at the beginning of the arrow will result in increases in the variable at the end of the arrow.

In the example presented in Figure 1, then, we represent a causal theory of population change. In the open-loop case, if we assume that birth rate increases, then, all else being equal, population increases (thus the positive sign in the causal link - arrow-between the two variables). Similarly, if we assume that birth rate decreases, then, all else being equal, population will decrease relative to what otherwise would have been. In this specific case, when birth rate declines, population might continue to grow but at a lower rate than before. In the first closed-loop case (Figure 1b), we can say that, as in the open-loop case, as birth rate increases, all else being equal, population increases and that, at the same time, as population increases, all else being equal, birth rate further increases creating a reinforcing feedback loop (denoted by an " $\mathrm{R}$ " in the loop identifier). Alternatively, if we first assume a decrease in birth rate, then population will decline bringing birth rate farther down, thus reinforcing the original change experienced.

A reinforcing feedback loop indicates the existence of a self-reinforcing (also called "positive") feedback process that reinforces growth (or decline) over time in the system in which it exists. A growing reinforcing feedback process, when left unchecked, will generate exponential growth patterns. In the second closed-loop case, a balancing (also called "negative") feedback process is depicted between population and death rate. In this case, first, a positive causal link between population and death rate is presented. If we assume an increase in population, as a result death rate will increase. In addition, in closing the feedback loop, a negative causal connection is depicted between death rate and population. As death rate increases, population decreases. A balancing feedback mechanism indicates the existence of a process that compensates changes in the system. The resulting behavior of a variable at any given point in time will depend on the different influences that such a variable has in a complex feedback set of interrelationships.

Figure 2 shows a causal-loop diagram that integrates the positive and negative feedback processes depicted in Figures 1b and 1c, thereby creating a set of causal loops with the potential to generate many different types of variable behavior depending on the relative strength of the influence of each loop. For example, if the reinforcing process between birth rate and population is increasing and stronger than the balancing process between population and death rate, then the over-time behavior of population will always be increasing. Alternatively, in order to create a stable population, a strong balancing process between population and death rate should exist. The feedback structure presented in Figure 2 has the potential to generate increasing, stable, and decreasing over-time behavior of its different variables (birth rate, population, and death rate), depending on the relative strength of the two feedback loops in it and on the initial conditions of the different variables. In causal-loop diagrams, feedback-based endogeneity becomes clear when a variable is depicted as part of a feedback loop or when, although not part of a feedback loop, it is a function of a variable that is part of a feedback loop.

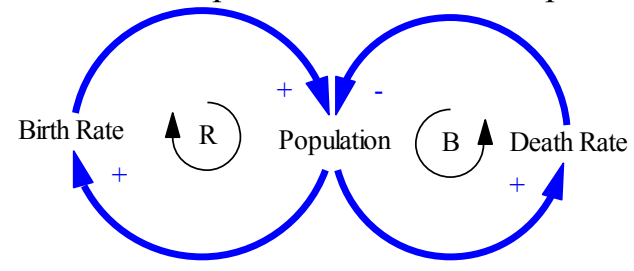

Figure 2: Multi-loop Causal Diagrams

While causal-loop diagrams are very useful in describing and increasing understanding of complexity in systems and in clarifying feedback-based endogeneity in such systems, they "do not distinguish between stocks and flows - the accumulation of resources in a system and the rates of change that alter those resources" (Sterman 2000, p. 140). Stock-and-flow diagrams can be used to explicitly represent ac- 
cumulations in the system and to clarify how such accumulations change as a function of their inflows and outflows - rates of change. Stock-and-flow diagrams are described next.

\subsection{Stock-and-flow Diagrams}

Stock-and-flow diagrams are useful in understanding complexity and feedback-based endogeneity in systems by clarifying the variables in the system, or model, that are stocks (accumulations) and by explicitly identifying the flows that change those stocks. Figure 3 shows a stock-and-flow diagram of the causalloop representation (presented in Figure 2) of the population theory discussed previously.

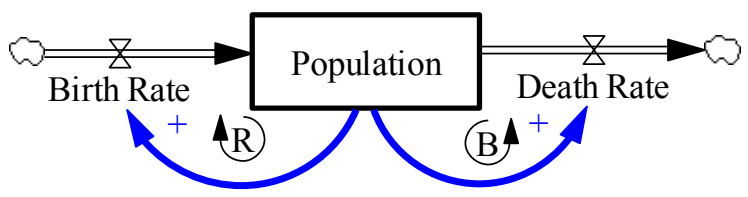

Figure 3: Stock-and-flow Diagram

In the system dynamics approach, stocks are always represented by rectangles (see population in Figure 3), while the flows that change a stock are represented by pipes pointing into (inflows) and out of (outflows) the stock. The flows are controlled by valves (see the inflow birth rate and the outflow death rate in Figure 3). Inflows add to stocks while outflows subtract from stocks. Although there is no theoretical limit to the number of inflows and outflows that a given stock can have, in order to maintain parsimoniousness, it is recommended to maintain these to as few as possible. In addition to stocks and flows, other variables may appear in a stock-and-flow diagram to complete the causal structure of the system, to close the feedback mechanisms that control the inflows and outflows linked to the stocks and to represent the exogenous variables needed. Finally, cloud-like icons at the end of the pipes represent the boundary of the model as sources and sinks for the flows. As such sources and sinks are outside the boundary of the model, they do not have any capacity limit and, therefore, do not in any way modify the resulting behavior of the variables in the model. If the capacity of a specific source or sink is particularly important in the model and can potentially change the behavior of the variables of interest, then this should be included in the model.

In the stock-and-flow version of the population model (represented in Figure 3), as in the causal-loop diagram of the model, there are two feedback loops: one reinforcing and one balancing ("R" and "B"). The reinforcing feedback loop connects population to birth rate using a causal link, and birth rate to population using a "pipe" or material link. In this case, as the pipe represents an inflow into the stock population, the polarity of the connection is positive, thus yielding a positive feedback process. The balancing feedback loop in the model is between population and death rate. Population is connected to death rate with a positive causal link, while death rate is connected to population through an outgoing flow. As death rate is an outflow from population, the connection is negative since death rate subtracts from population. Identifying the balancing loop population-death rate-population can be tricky as the negative causal connection between population and death rate is not completely explicit in this type of diagramthere is no directed arrow connecting death rate and population, this is implied.

As in causal-loop diagrams, in stock-and-flow diagrams, feedback-based endogeneity becomes explicit when a variable is depicted as part of a feedback loop, or when, although not a part of a feedback loop, it is a function of one. To exemplify the differences between how endogeneity and feedback are represented in system dynamics models and in ABMs we will develop a model using both approaches (for an example of the development of a model using both the system dynamics and agent-based approaches for the purpose of testing model reproducibility, see North and Macal 2009). In this paper, we will use a model from epidemiology - the SIR (Susceptible-Infectious-Recovered) Model-to compare the modeling approaches (for a detailed study contrasting the two approaches, see Rahmandad and Sterman 2008). 
Additionally, we will clarify how we think agent-based modelers may be able to explicitly talk about endogeneity in their models by using tools from the system dynamics approach presented earlier.

\section{SYSTEM DYNAMICS SIR MODEL}

In this section we describe an SIR model using the system dynamics approach (the equations of the model are available from the authors). In this model, we consider an aggregate approach to represent the three key populations that lead to the progression of an epidemic: susceptible, infectious, and recovered. In Table 2, we present a model-boundary chart of the model. Here, the endogenous, exogenous, and excluded variables are listed. In order to create such a list, the modeler must have an understanding of the system under study through a set of equations or a set of pre-identified relationships that capture model interdependencies. A model-boundary chart, although useful for the modeler, is generally produced to help communicate model components to an audience that is not familiar with the details of the model.

Table 2: Model-boundary Chart of the System Dynamics SIR Model

\begin{tabular}{lll}
\hline Endogenous & Exogenous & Excluded \\
\hline Susceptible Population (S) & Contact Rate (c) & Immunization Rate \\
Infectious Population (I) & Infectivity (i) & Recidivism Rate \\
Recovered Population (R) & Average Recovery Time (d) & Death Rate \\
Infection Rate & Total Population (N) & \\
Recovery Rate & Initial Infectious Population & \\
\hline
\end{tabular}

In this model, five variables will be endogenously computed using five exogenous variables. As stated before, although the list of excluded variables can be infinite, the idea is to list the variables that are purposefully excluded from the model in order to make it parsimonious. The list of excluded variables, therefore, helps clarify the boundary of the model by providing a list of variables (relevant to the problem) that are not part of the model. In addition to stating the limits of the model, the list of excluded variables provides a clear path forward for future development or expansion of the model. The variables listed in a model-boundary chart need not be exhaustive, especially in the case of large models. However, it is important to include all the variables of interest that will allow the modeler and the modeler's audience to understand the critical elements in the model.

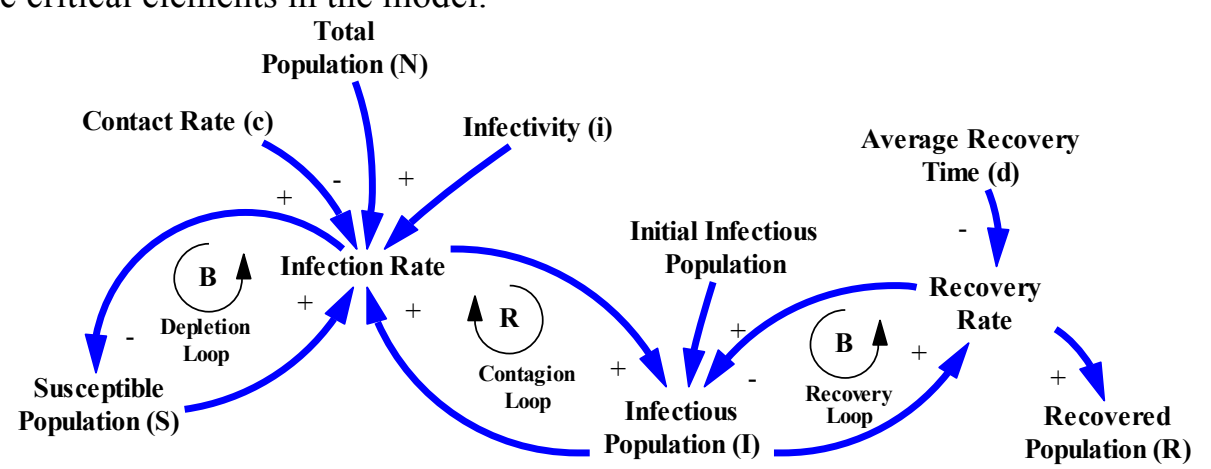

Figure 4: Feedback Structure of the SIR System Dynamics Model (causal-loop diagram)

Figure 4 shows a casual-loop diagram of the SIR system dynamics model used as an example. In this figure, the 10 variables of the model are shown using causal links to identify the way in which the variables interact with each other, resulting in three feedback mechanisms: the depletion loop (balancing), the contagion loop (reinforcing), and the recovery loop (balancing). The variables that are part of these feedback loops are endogenously computed in the model and, therefore, the resulting behavior is, at least partially, a function of a previous value of the same variable. Although not needed to discover the interde- 
pendencies present in the model (as these are known a priori), using a causal diagram to depict the interactions of the variables clarifies its underlying feedback structure.

Figure 5 shows a stock-and-flow diagram of the SIR model. In this diagram, both the causal loops and the stocks and flows are explicit allowing the modeler to visually represent material flows in the model (elements flowing through the pipes in and out of the stocks in the model) and to see how these interact through feedback processes (although the relationships are included in the model's design). By inspecting this diagram, the possible flow from susceptible to infectious (infection rate) and from infectious to recovered (recovery rate) are salient. In this small sample model, this progression might be intuitive to many; however, in larger models, the progression through the different stages might not be easily discerned directly from equations or other type of diagrams and representations. In order to avoid unmanageably large diagrams, in the case of extremely large detailed models, stock-and-flow diagrams should be focused on depicting the dynamic complexity of the model, not its detail complexity (for a description of the concepts of dynamic and detail complexities, see Senge 1990).

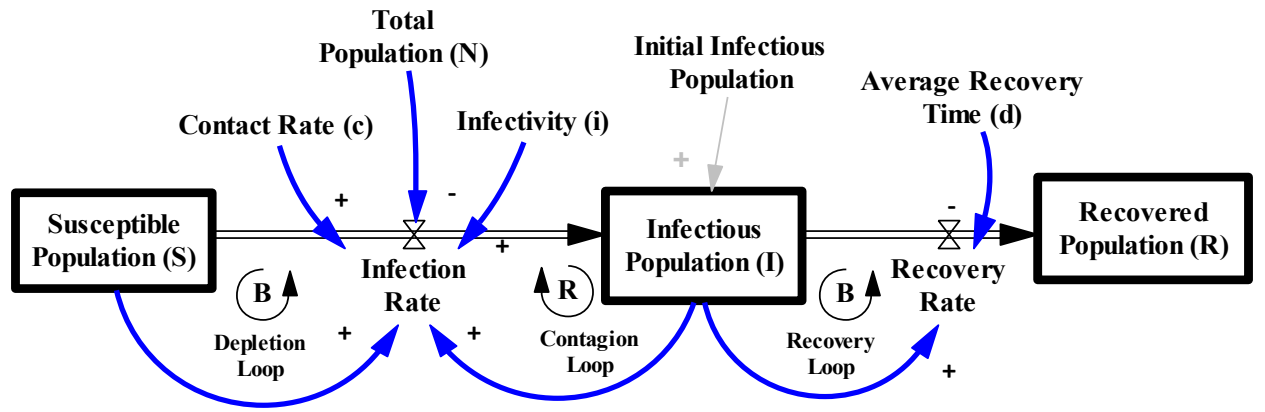

Figure 5: Feedback Structure of the SIR System Dynamics Model (stock-and-flow diagram)

The model described in the previous section generates the behavior shown in Figures $6 \mathrm{a}$ and $6 \mathrm{~b}$. The resulting behavior is a function of the different exogenous parameters used and the feedback process influencing the susceptible, infectious, and recovered populations. One can see that the behavior represents aggregate, deterministic, results of an epidemic process. The infectious population peaks at around day 20 slightly below 100 people, while the whole epidemic process unfolds over an 80-day period.

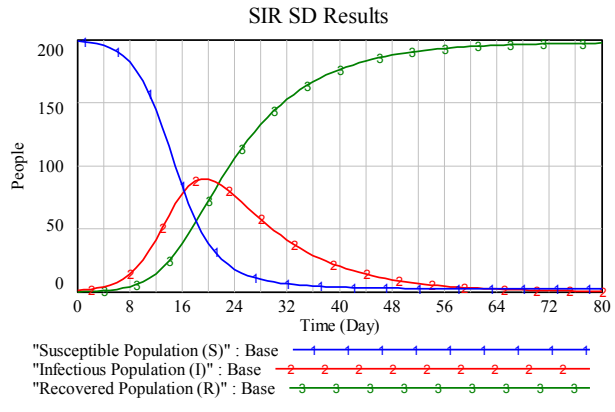

(a)

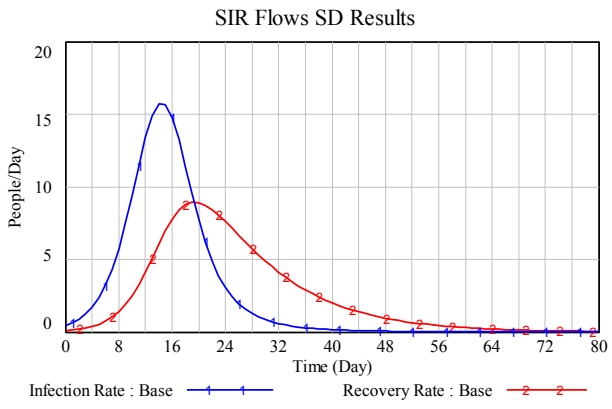

(b)

Figure 6: Resulting Behavior of the System Dynamics Version of the SIR Model. (a) Stocks. (b) Flows

\section{AGENT-BASED SIR MODEL}

In this section, we describe an agent-based implementation of the SIR Model in which each individual in the population is individually represented. In the following description, we specify the model in pseudocode and take an object-oriented programming approach, in which agent types are defined as classes (Macal 2010). Agent interactions are specified by methods defined at the class level. Methods operate on the agent instances in the corresponding agent classes to model agent interactions (in the case of the SIR 


\section{Martinez-Moyano and Macal}

model, agent interactions are contacts between agents) and to update the agent states through time (model code is available from the authors).

In the model, we define a single agent class in pseudo-code that covers any individual in the population:

Agent_Class = agent[agent_unique_identifier, agent_disease_state, time_of_recovery (if agent is in disease state "I" or " $\mathrm{R}$ ", if agent is in disease state " $\mathrm{S}$ " is $\infty$ )]

The state of an agent at any time is its particular disease state. The agent state is the only information in the model that is endogenously updated as all other parameters are constants. The number of individuals in the population (in each disease state) is implied by the number of agents (in each disease state). In agent-based models, state diagrams (not shown here) can be used to show how an agent moves between states over time in response to endogenous and/or exogenous influences (North and Macal 2007). Based on the agent class definitions above, there are $\mathrm{N}$ agent instances in various disease states of $\mathrm{S}, \mathrm{I}$, and $\mathrm{R}$, where $\mathrm{N}$ is the total population size. In the process for updating the agent states, the interaction between agents and agent states is depicted. The logic within the update method is shown in Figure 7a, and the resulting behavior is shown in Figure 7b. The resulting behavior (especially the thick lines in Figure $7 \mathrm{~b}$ representing the average of the stochastic results) of the agent-based implementation is comparable to the results of the system dynamics version of the model (see Figure 6a). Notice that in Figure 6b, the behavior of the flows between stocks in the system are explicitly identified and presented. Normally in agent-based models, no flows are computed due to the level of granularity of computations performed (agent-to-agent interactions) and due to the inexistence of explicit accumulators for agents that share the same disease state.

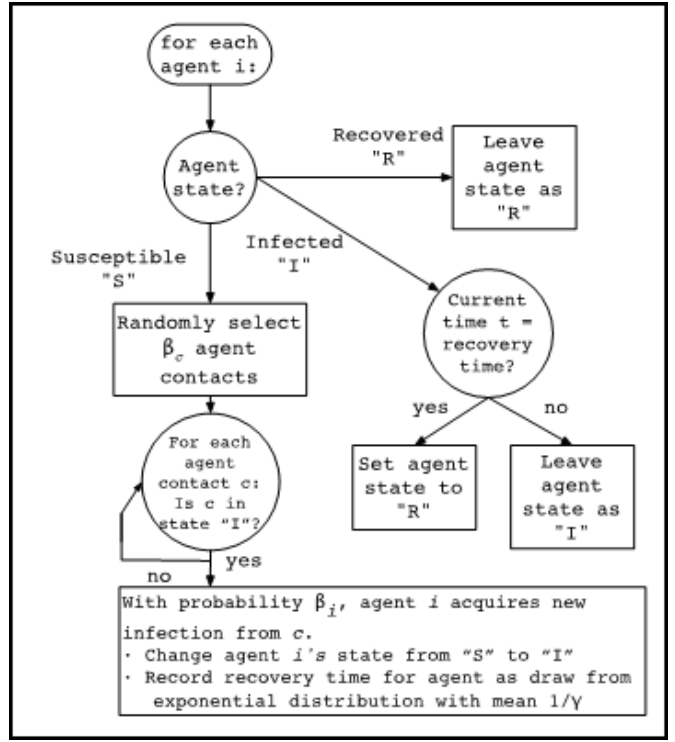

(a)

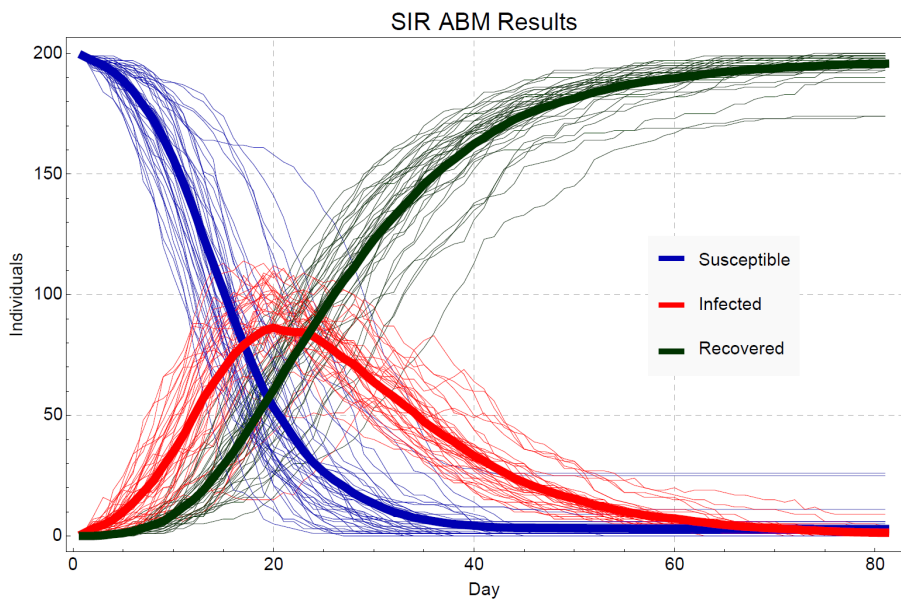

(b)

Figure 7: (a) Logic of the ABM for Updating the Agent Disease States at Each Time Step. (b) Resulting Behavior of the Agent-based Version of the SIR Model.

\section{REPRESENTING FEEDBACK AND ENDOGENEITY IN AGENT-BASED MODELS}

As described in the previous section, agents' disease states are updated every time step following the logic described in Figure 7a. To identify, understand, and explicitly represent the flow of agents from one disease state to the next it is crucial to make explicit the interactions among agents (and the endogeneity present in the model) as it is in the mechanisms underlying the flow of agents from one disease state to 
the next where endogeneity resides. In order to explicitly depict the interactions among agents, and to make feedback-based endogeneity in agent-based models more salient, we propose the use of agent feedback diagrams to bridge the typical logic-based representation of agent-based models with a more feedback-oriented representation (e.g., causal-loop or stock-and-flow representations of model structure).

In the agent feedback diagram shown in Figure 8, we propose an explicit representation of the interaction of agents (see the interaction of agents in the infectious and susceptible states). Also, we show the physical flow of agents from one disease state to another at different time steps in the simulation (the thick arrows represent the flow of agents from one disease state to another). Agents in the susceptible state can stay in that state or go to the infectious state (depending on the result of their interaction as a function of contact and infectivity levels represented by $c$ and $\beta$ in the diagram), while agents in the infectious state can stay there or move to the recovered state when their recovery time has elapsed. Finally, agents in the recovered state can only stay there as recovered is an absorbing state in the system. From an agent feedback diagram with explicit time representation (such as the one shown in Figure 8), modelers may then move closer to a stock-and-flow-like representation of their models by collapsing all the different points in time represented in such diagram into a single representation. However, if the agent behaviors include significant nonlinearities or discontinuities, then collapsing the time dimension in the diagram may become difficult or unlikely. When a representation without the time dimension is possible, the underlying flow of agents between disease states at any point in time is depicted (for an example see Figure 9).

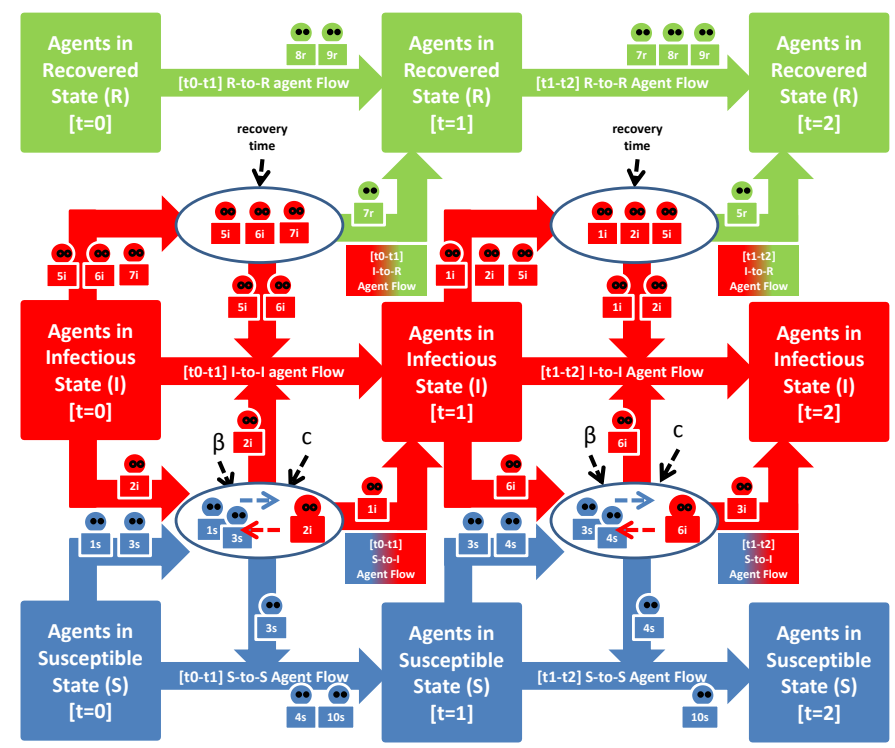

Figure 8: Agent Feedback Diagram (with explicit representation of time)

In an agent feedback diagram without explicit representation of time (as shown in Figure 9), we show the information needed to compute the disease state of agents over the simulated time- arrows with dotted lines represent information about the disease state of the interacting agents. For example, in order to compute agent states for agents in the susceptible disease state, information about the current disease state is needed and information about the disease state of agents with which the agents in the susceptible state are in contact (i.e., information about agents in the infectious state). For agents in the infectious state, information about their previous disease state and information about contacts with agents in the susceptible state are needed to compute their new disease state. Representing the information exchange in this way allows the endogenous nature of the logic to become explicit and easier to identify.

Summarizing, in the agent feedback diagram shown in Figure 9, we represent the flow of agents (thick, solid arrows) through the different disease states (susceptible, infectious, and recovered) and the 
information needed (thin, dotted arrows) for the flows to be identified and computed making the feedback structure of the model explicit and salient.

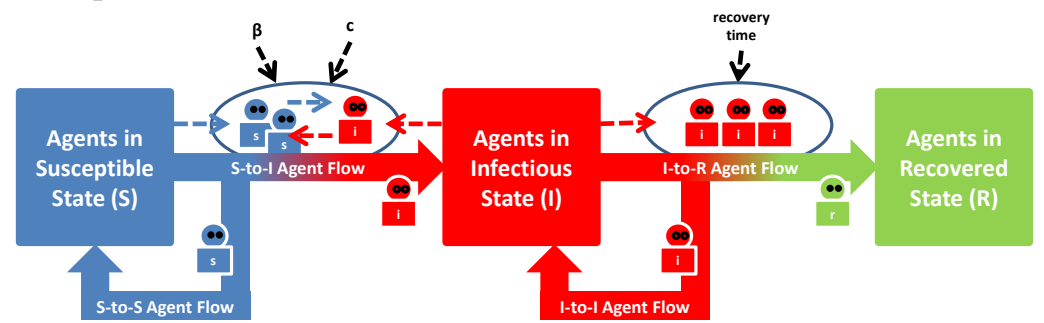

Figure 9: Agent Feedback Diagram (without explicit representation of time)

The agent feedback diagrams presented in Figures 8 and 9 can be used as bridges between the typical logic-based depictions of agent models (such as the one presented in Figure 7a) and feedback-oriented representations such as causal-loop or stock-and-flow diagrams characteristically used in the system dynamics approach.

\section{CONCLUSIONS}

This paper describes how feedback-based endogeneity in ABMs can be identified, described, and emphasized to increase the explanatory power of ABMs. Additionally, concepts and insights from system dynamics modeling are used and applied to agent-based modeling and simulation. By advancing a distinction between structural endogeneity and behavioral endogeneity in models, we strive to improve how to communicate endogeneity-related ideas in both the system dynamics and the agent-based paradigms. Also, we propose the use of the derived agent feedback diagrams and diagrams commonly used in system dynamics (model boundary charts, causal-loop diagrams, and stock-and-flow diagrams), additionally to the state and logic-flow diagrams from agent-based modeling, to communicate the underlying structure of agent-based models-making the endogenous mechanisms included in such models explicit and salient. Furthermore, since the identification of the degrees of structural and behavioral endogeneity and the development of agent feedback diagrams is potentially very labor intensive for non-trivial agent-based models (typically including multiple agent classes), the development of automated computational approaches to identify endogeneity in agent-based models and to generate their associated agent feedback diagrams seems warranted.

\section{ACKNOWLEDGMENTS}

This manuscript has been created in part by UChicago Argonne, LLC, Operator of Argonne National Laboratory ("Argonne"). Argonne, a U.S. Department of Energy Office of Science laboratory, is operated under Contract No. DE-AC02-06CH11357. The U.S. Government retains for itself, and others acting on its behalf, a paid-up nonexclusive, irrevocable worldwide license in said article to reproduce, prepare derivative works, distribute copies to the public, and perform publicly and display publicly, by or on behalf of the Government.

\section{REFERENCES}

Antonakis, John, Samuel Bendahan, Philippe Jacquart, and Rafael Lalive. 2010. "On making causal claims: A review and recommendations." The Leadership Quarterly no. 21 (6):1086-1120. doi: 10.1016/j.leaqua.2010.10.010.

Auchincloss, Amy H., and Ana V. Diez Roux. 2008. "A New Tool for Epidemiology: The Usefulness of Dynamic-Agent Models in Understanding Place Effects on Health." American Journal of Epidemiology no. 168 (1):1-8. doi: 10.1093/aje/kwn118. 
Forrester, Jay W. 1968. Principles of Systems. Cambridge MA: Productivity Press.

Forrester, Jay Wright. 1958. "Industrial Dynamics: A Major Breakthrough for Decision Makers." Harvard Business Review no. 36 (4):37-66.

Forrester, Jay Wright. 1961. Industrial Dynamics. Cambridge MA: Productivity Press.

Macal, Charles M. 2010. To Agent-based Simulation from System Dynamics. In Winter Simulation Conference, edited by B. Johansson, S. Jain, J. Montoya-Torres, J. Hugan and E. Yucesan. Baltimore, MD: IEEE.

Mojtahedzadeh, Mohammad, David Andersen, and George P. Richardson. 2004. "Using Digest to implement the pathway participation method for detecting influential system structure." System Dynamics Review no. 20 (1):1-20. doi: 10.1002/sdr.285.

Nakamura, Alice, and Masao Nakamura. 1998. "Model specification and endogeneity." Journal of Econometrics no. 83 (1-2):213-237. doi: http://dx.doi.org/10.1016/S0304-4076(97)00070-5.

North, Michael J., and Charles M. Macal. 2007. Managing Business Complexity: Discovering Strategic Solutions with Agent-based Modeling and Simulation. New York, NY: Oxford University Press.

North, Michael J., and Charles M. Macal. 2009. "Agent-based Modelling and Systems Dynamics Model Reproduction." International Journal of Simulation and Process Modelling no. 5 (3):256-271.

Oliva, Rogelio. 2004. "Model structure analysis through graph theory: partition heuristics and feedback structure decomposition." System Dynamics Review no. 20 (4):313-336. doi: 10.1002/sdr.298.

Rahmandad, Hazhir, and John Sterman. 2008. "Heterogeneity and Network Structure in the Dynamics of Diffusion: Comparing Agent-Based and Differential Equation Models." Management Science no. 54 (5):998-1014. doi: 10.1287/mnsc.1070.0787.

Richardson, George P. 1991. Feedback Thought in Social Science and Systems Theory. Second Edition ed, System Dynamics Series. Waltham, MA: Pegasus Communications.

Richardson, George P. 2011. "Reflections on the foundations of system dynamics." System Dynamics Review no. 27 (3):219-243. doi: 10.1002/sdr.462.

Richardson, George P., and Alexander L. Pugh, III. 1981. Introduction to System Dynamics Modeling with DYNAMO. Cambridge MA: Productivity Press.

Senge, Peter M. 1990. The Fifth Discipline: The Art and Practice of the Learning Organization. New York, NY: Doubleday/Currency.

Sterman, John D. 2000. Business Dynamics: Systems Thinking and Modeling for a Complex World. Boston, MA: Irwin McGraw-Hill.

\section{AUTHOR BIOGRAPHIES}

IGNACIO J. MARTINEZ-MOYANO is a Computational Social Scientist with the Decision and Information Sciences Division of Argonne National Laboratory and a Research Fellow at the Computation Institute of The University of Chicago. His research focuses on the application and theoretical development of system dynamics modeling and computer simulation to advance the theories and understanding of human behavior. He has published in academic journals such as Organization Science, the Journal of Public Administration Research and Theory, Transactions on Modeling and Computer Simulation, Computers \& Security, and the System Dynamics Review. His e-mail is imartinez@anl.gov.

CHARLES M. MACAL, PhD, PE, is the Director, Center for Complex Adaptive Agent Systems Simulation $\left(\mathrm{CAS}^{2}\right)$, Argonne National Laboratory. He is a member of the INFORMS-Simulation Society, Association for Computing Machinery, the Society for Computer Simulation International, and the System Dynamics Society. He is on the editorial boards of Transactions on Modeling and Computer Simulation, Simulation, and Complex Adaptive Systems Modeling. He has a Ph.D. in Industrial Engineering \& Management Sciences from Northwestern and a Master's Degree in Industrial Engineering from Purdue. Contact: macal@anl.gov. He is a registered professional engineer in the State of Illinois. 